\title{
Reconocimiento de la familia del terapeuta: construcción de su identidad y prácticas
}

\section{Re-knowledge of the therapist's family: construction of their identity and practices}

\author{
Lucía Pérez Sánchez y Marcela Rábago de Ávila \\ Universidad Autónoma de Nayarit, Mexico \\ "El terapeuta a lo largo de su vida, va construyendo referentes conceptuales que cons- \\ tituirán su realidad teórica-profesional o estilo terapéutico. Se ubicará ante su elección, \\ con toda la carga de su propia historia, como portavoz de un código familiar” ...
}

(Ceberio, Moreno y Des Champs, 2000)

\begin{abstract}
Resumen: El siguiente trabajo es el resultado del análisis y revisión llevada a cabo posterior a una intervención clínica sistémica, con familias con un adulto mayor con diabetes tipo 2 . A través del método cualitativo, partiendo del diseño fenomenológico con base en estudio de caso. El objetivo fue explorar y describir los constructos personales que cada terapeuta construye a partir de la experiencia de su práctica, y la influencia de su familia de origen. A través del uso de la técnica de la rejilla de Kelly, se obtuvieron los siguientes constructos personales como principales resultados: disposición, cuidado, empatía, sensibilidad y participación como las características más destacadas para lograr optimizar los recursos del terapeuta. Los constructos personales atraviesan de manera importante la forma en que definen sus experiencias con su familia de origen, y con las de su práctica profesional, así también se expone cómo el encuentro de la familia y el terapeuta nunca es pasivo, siempre existe una transformación mutua. Por tanto, la familia de origen del terapeuta se ve como un recurso para la autorreflexión de la práctica profesional, a través de la cual organiza su mundo de experiencia y su propio estilo terapéutico.

Palabras claves: Papel de la familia, experiencia personal, resonancias, constructos personales, y psicoterapia.
\end{abstract}

Abstract: The purpose of this study was to analyze the experience of the clinical work of two therapists and to know the perception, resonances, and personal constructs that guided their clinical practice and how they built their personal world around such experience.

La correspondencia sobre este artículo debe enviarse a la primera autora al E-mail: lucia@systemica.com.mx

(cc) BY-NC-ND Este es un artículo Open Access bajo la licencia CC BY-NC-ND. 
Using a qualitative method of constructivist design and Kelly repertory grid technique, the following constructs were obtained as main results: disposition, care, and empathy as the most outstanding characteristics to achieve optimization of family resources in a clinical work. This will make no sense if not congruent in the personal life of the therapist. The best impact on intervention is achieved through being authentic. In addition, identification with the therapist's own family of origin played a crucial role in the professional development and empathy of therapists. The family of origin is seen as a resource for self-reflection of professional practice and will help identify the personal constructs through which therapists organize their world of experience and own therapeutic style.

Keywords: Role of the family, personal experience, resonances, constructs and psychotherapy.

La familia es parte del ser humano desde el día de su nacimiento hasta su muerte. Es en este contexto donde se generan toda una serie de procesos psicológicos necesarios para el crecimiento y desarrollo del individuo, por lo que la familia de origen del terapeuta no podrá ser la excepción.

Framo (1996) valora la necesidad de incluir en el trabajo terapéutico a la familia de origen del terapeuta, pues en ella se configura la percepción de realidad y las maneras de relaciones sociales de la persona, incluyendo el espacio terapéutico. También considera que las fuertes tensiones que experimenta el terapeuta familiar se sostienen en la conciencia que tiene de sí mismo, y en la elaboración de temas relevantes con su propia familia de origen.

Por esta razón, con frecuencia el terapeuta puede observar en la clínica, familias con problemas o enfermedades similares a las de su propia familia de origen. Estos aspectos de la vida que experimentan los terapeutas al trabajar con personas con padecimientos, que en sus respectivas familias también están presentes, en ocasiones no se les asigna valor, manteniéndolos al margen, y suele costar mucho trabajo su identificación (White, 2011).

Por lo que, la importancia de revisar las resonancias y las emociones referidas a la familia del terapeuta a través de la autorreflexión, se convierten en parte de las experiencias más significativas en la vida de los y las psicoterapeutas, y con ello la mejor herramienta en el trabajo clínico (Canevaro, et al., 2016; Hernández, 2012; Louro, 2003).

En síntesis, explorar las historias y narraciones de vida familiar del terapeuta y la riqueza de saberes que provienen de ella, contribuye significativamente para que se logren experimentar como personas y profesionales preparadas y capaces (White, 2011; 2002).

Con base a lo anterior, se llevó a cabo el presente trabajo, derivado de una intervención clínica sistémica, que tenía por objetivo el abordaje familiar para el mejoramiento en el cuidado y atención de un adulto mayor con diabetes tipo 2. Al término de las sesiones que conformaron el tratamiento, se analizaron las resonancias que tuvieron las terapeutas a cargo de estas familias, y así poder conocer de qué manera su actuación clínica llegó a tener impacto en la vida de ellas.

Para esto, se utilizó un cuestionario con preguntas abiertas, de tal manera que las respuestas vertidas, ayudarían al proceso de autorreflexión de las terapeutas. También se utilizó un instrumento denominado rejilla de Kelly que evalúa la estructuración y significado que el sujeto da a una experiencia en sus propios términos. La técnica de la rejilla es una manera en particular de llevar a cabo una entrevista estructurada, acorde a los objetivos definidos por el investigador, con el fin de obtener y analizar los constructos personales, derivados del análisis de las propias palabras del entrevistado (Feixas y Cornejo, 1996).

\section{Método}

La presente investigación es el resultado de la revisión llevada a cabo posterior a una intervención clínica, en familias con un adulto mayor con diabetes mellitus tipo 2. A través del método cualitativo, partiendo del diseño fenomenológico con base en estudio de caso. Con el objetivo de explorar y describir la experiencia desde la 
propia subjetividad de las terapeutas y los respectivos constructos personales que cada una de ellas construye con base a dicha experiencia (Denzin y Lincoln, 2015; Hernández, Fernández y Baptista, 2014; Andersen, 2005; Latorre, Del Rincón y Arnal, 1996).

\section{Objetivo}

Analizar la experiencia del trabajo terapéutico que vivieron las terapeutas a cargo de una intervención familiar, y conocer su percepción, resonancias, y constructos personales que guiaron su práctica clínica, así como, la construcción de su mundo personal en torno a dicha vivencia (Pakman, 1999).

Objetivos específicos. Los objetivos específicos fueron:

- Analizar los constructos personales de cada terapeuta, respecto a su experiencia de vida con un familiar adulto mayor con diabetes tipo 2.

- Analizar la resonancia de la interacción con un adulto mayor, en sus propios procesos de envejecimiento.

- Conocer cómo consideran ellas que puede ser una intervención ideal, dada las circunstancias de las familias con las que llevaron a cabo la intervención.

\section{Instrumentos}

Entrevista para la elaboración de la rejilla de Kelly (1955). Para la presente intervención se aplicó la técnica de rejilla, por ser el instrumento por excelencia en la evaluación constructivista (Neimeyer,1996; Neimeyer y Mahoney, 1998).

Cuestionario abierto denominado "Formato de retroalimentación para el equipo de trabajo". Este cuestionario fue diseñado específicamente para la autoevaluación de la intervención familiar.

Ambos instrumentos fueron suministrados por una tercera persona, investigadora colaboradora en este trabajo específico, la cual es doctora en psicología y está capacitada en la utilización de la rejilla de Kelly en la evaluación psicoterapéutica, además de no haber estado previamente como partícipe de la investigación que dio origen a este trabajo.

Participantes. Dos terapeutas responsables del trabajo clínico con familias con un adulto mayor con diabetes tipo 2, que además también tienen un familiar con este padecimiento.

\section{Procedimiento}

Posterior a la culminación de la intervención familiar, se entregó el formato de retroalimentación a cada terapeuta para ser contestado. Los tópicos que abordó este cuestionario fueron: observaciones respecto a las actividades realizadas, experiencia vivida con relación a la intervención, resonancias con mi propia vida, resonancias con mi propio proceso de envejecer y resonancias en mi interacción con mis propios familiares adultos mayores. En otra sesión, se llevó a cabo la entrevista para elaborar la rejilla de Kelly, su aplicación tuvo una duración aproximada de 70 minutos. Para la triangulación de la información de los instrumentos, se procedió a realizar el análisis de la rejilla (a continuación, se explica su procedimiento a detalle) y los constructos personales sirvieron de filtro para la revisión del contenido escrito en el formato de retroalimentación.

En cuanto a la técnica de la rejilla se sometió a un análisis matemático que exploró de manera confiable cuantitativa y cualitativamente los constructos de cada una de las terapeutas de la presente investigación. Dichos constructos son de naturaleza bipolar y se organizan jerárquicamente (Feixas y Cornejo, 1996).

Esto permitió analizar los constructos personales de cada una de las terapeutas como resultado de la entrevista, en la cual terapeuta y entrevistador fueron construyendo la rejilla con base a las respuestas. Por lo que, partiendo de la experiencia personal del contexto familiar de las terapeutas y su experiencia clínica, y 
con base a los objetivos del investigador, fueron seleccionados los siguientes elementos: yo, yo ideal, intervención, familia con la cual se realizó la intervención, adulto mayor, proceso de envejecimiento personal, familiar adulto mayor, habilidades terapéuticas, intervención ideal, y persona no grata que pudo haber existido en la intervención al obstaculizar la misma.

Los elementos fueron presentados en diadas a cada una de las terapeutas de manera individual y se preguntó sobre las diferencias o semejanzas entre ambos elementos, y así sus respuestas generaron los constructos personales de cada una. Los criterios que se tomaron en cuenta en la presentación de las diadas de elementos son los siguientes:

1. Cada elemento debió presentarse por lo menos una vez en las diadas presentadas a las terapeutas.

2. El elemento YO debió ser presentado con la mayoría de los elementos de las diadas mostradas.

A continuación, se procedió a realizar la puntuación de cada uno de los elementos con relación a los constructos que elaboraron cada una de las terapeutas, y a la hora de puntuar se incluyó el YO IDEAL, debido a que esto es un requisito para la calificación en la página web que analiza los resultados de la rejilla, la puntuación fue en una escala de 1-7.

Una vez que fueron construidas cada una de las rejillas, se procedió a ingresar los datos al sitio web http:// www.tecnicaderejilla.net/programa para el análisis matemático con el programa RECORD versión 5.0. Esta es una página web especializada en el procesamiento de resultados en la aplicación de la técnica de la rejilla de constructos personales de Kelly. Dicho programa calcula los índices cognitivos globales: Intensidad, potencia discriminativa y polarización.

El Índice de Polarización mide el modo radical en que el sujeto percibe un constructo. Las puntuaciones altas son consideradas como una forma de rigidez cognitiva, tomando en cuenta que para ser una puntuación alta deberá estar por encima de la puntuación obtenida en la polarización total.

El Índice de Intensidad es indicador de la centralidad del constructo, por lo que, el que presenta mayor intensidad es el que tiene más correlación con el resto de los constructos debido a su centralidad. El constructo con más baja intensidad es el más desligado y periférico (Feixas y Cornejo, 1996).

La Potencia Discriminativa mide la flexibilidad en la aplicación de los constructos; por lo que los constructos que obtienen mayor puntuación en potencia discriminativa nos indican que son constructos más flexibles (Feixas y Cornejo, 1996).

La triangulación de los resultados se llevó a cabo con el análisis del formato de retroalimentación, al que posterior a la intervención dieron respuesta las terapeutas, en donde dan testimonio de sus experiencias personales. Una vez aplicado el formato de retroalimentación, se solicitó a las terapeutas su apoyo para la construcción de la rejilla de constructos personales respecto a su experiencia clínica, y con la de su propia familia de origen, lo cual les otorga una poderosa autentificación de los saberes y habilidades como terapeutas que contribuye a que sus vidas y su práctica sean densamente enriquecedoras, porque la revisión y reflexión de sus resonancias puede resultar reconfortante, incluso de manera trascendental, no solo en lo profesional sino en la vida cotidiana con sus familias de origen (White, 2002).

De los resultados obtenidos en la rejilla aplicada a cada una de las terapeutas respecto al índice de polarización (rigidez cognitiva); índice de intensidad (centralidad del constructo) y potencia discriminativa (flexibilidad en la aplicación del constructo), fueron seleccionados los constructos con mayor puntuación en cada uno de los indicadores. La triangulación de los resultados se obtuvo al llevar a cabo el análisis de los resultados de la aplicación de la rejilla y su contrastación con los resultados de la entrevista aplicada a las terapeutas.

\section{Resultados}

\section{Resultados de la primera terapeuta}

Constructos personales. En la tabla 1 se muestran los resultados que se obtuvieron de la primera terapeuta, cabe señalar que fueron elegidos los de mayor puntaje en cada uno de los indicadores. 
Índice de Polarización. A continuación, se presentan los resultados de los puntajes más altos obtenidos en los constructos del índice de polarización, el cual hace referencia a la rigidez cognitiva: Desunión - unión familiar: 70; Apatía - empatía: 60; Descuido - cuidado 50. Al ser constructos de difícil modificación, en la práctica terapéutica se pueden ver traducidos y observables específicamente en la importancia y trascendencia que para ella tiene el hecho de ser cuidadosa no solo con su intervención, sino también el cuidado y compromiso que adquiere con las personas que han confiado en ella para apoyarlos en la resolución del problema que los llevan a consulta.

De igual manera, esta terapeuta considera que a través de la empatía se logra conectar su sentido de ser terapeuta con las necesidades de la familia y con ello, se consigan solventar los conflictos, y hacer frente en este caso de manera conjunta a la diabetes. En definitiva, dicho afrontamiento, desde el punto de vista del terapeuta, no puede ser dado sin el apoyo constante y compromiso que surge de la unión familiar, para encarar a las situaciones que se presentan como consecuencia de la enfermedad.

Índice de Intensidad. Respecto a los puntajes más altos del índice de intensidad el cual hace referencia a la centralidad de dichos constructos, es decir, la mayoría de los demás constructos elaborados girarían en torno a ellos, los resultados son los siguientes: Debilidad - fuerza: .43; Cerrado - disposición: .40; Apatía-empatía: .40. Es decir, para esta terapeuta es de suma importancia tanto en su vida personal como profesional mantener una apertura y buena disposición para los cambios, aun cuando ello implique que el cambio no sea favorable, como lo es en el curso de la vida de una persona con diabetes, pero que con esta actitud de apertura a los nuevos eventos en la vida y permite llevar acabo la exploración de nuevos caminos, se genera de manera indiscutible un incremento en las posibilidades de enfrentar a la enfermedad de manera más efectiva y satisfactoria. Sin duda, todo ello es parte de las fortalezas de la terapeuta, no solo en su vida personal, sino en su práctica y acompañamiento en las consultas a su cargo.

Para ella, hacer frente a los avatares del destino es una muestra de la fortaleza de su ser y hacer como terapeuta. De nueva cuenta, la empatía al ser un constructo central en su vida, éste se traduce en su capacidad para ponerse en el lugar del otro, lo cual no solo facilita su labor terapéutica, sino también, ser vista como una persona que garantiza contar con su apoyo.

Potencia Discriminativa. Respecto a la potencia discriminativa que hace referencia a la flexibilidad en la aplicación de dichos constructos, los que obtuvieron las puntuaciones más altas fueron: Frustración - satisfacción: 3. Para esta terapeuta, la importancia radica en llevar a cabo una práctica terapéutica capaz de colaborar de manera empática con las personas, lo cual se hace evidente no solo en la satisfacción de aquellos que asisten con ella para solicitar sus servicios, sino también en satisfacción personal como en este caso, de haber logrado colaborar de manera conjunta con las familias con un adulto mayor con diabetes.

En cuanto a estos constructos personales descritos, la conexión con el legado de su familia de origen y el haber tenido la experiencia de vivir de cerca con un familiar adulto mayor con diabetes tipo 2, vuelven a confirmar que la empatía, el cuidado y la unión familiar son valores que atravesaría su práctica clínica y a su vez se verán reflejadas en futuras intervenciones con familias que tienen un adulto mayor con alguna enfermedad crónico-degenerativa. Pues como ella misma describe, la importancia que significó en este trabajo terapéutico su conexión con sus abuelas, las cuales padecieron diabetes muchos años de su vida, por lo que estuvo involucrada en cómo es la enfermedad, cuáles son los síntomas, tratamiento, y sobre todo las dificultades que conlleva, si no existe un control adecuado.

Al tener una referencia tan cercana con su familia de origen y la enfermedad, la ayudó a considerar la vía de la prevención como un recurso imprescindible. Lo que le brindó la oportunidad para valorar y analizar su propia interacción con sus familiares mayores, en especial recordó a su abuela paterna, identificándola con uno de los adultos mayores que estuvieron bajo su atención. Este adulto en particular, al que hace referencia, su mayor demanda era con respecto a un gran sentimiento de soledad, y al igual que él, ella recuerda que su abuela vivía sola, y así era como ella se sentía. Por tal motivo, al escuchar las peticiones o reclamos de esta familia le fue fácil sentir empatía por las dos partes, el familiar mayor y los familiares que lo cuidaban. El adulto mayor le recordó la soledad que sentía su abuela y su vulnerabilidad que percibía al vivir sola, y al mismo tiempo la sensación de culpa y frustración por parte de la familia, de no haber podido complacer esta petición de manera satisfactoria. 
Por lo que, este episodio familiar de la terapeuta se convierte en un arsenal de herramientas que le darían una gran variedad de posibles acciones profesionales, pero a la vez se convierte en un espejo retrovisor que le amplía el campo de visión y le muestra de manera más clara el espacio que requiere observar. A continuación, se describe cómo sería para ella, una intervención ideal.

Tabla. 1. Constructos e indicadores de la primera terapeuta

\begin{tabular}{lccc}
\hline Constructos & Polarización & Intensidad & Potencia discriminativa \\
\hline Cerrado-disposición & 40 & .40 & 2.50 \\
Aislamiento-intimidad & 20 & .26 & 1.60 \\
Debilidad-fuerza & 20 & .43 & 1.20 \\
Descuido-cuidado & 50 & .25 & 2.50 \\
Resistencia-abierto & 10 & .29 & 2 \\
Depresión-ánimo & 40 & .38 & 2 \\
Racionalización-emoción & 20 & .11 & 2.40 \\
Apatía-empatía & 60 & .40 & 1.20 \\
Desunión-unión familiar & 70 & .30 & 1.20 \\
Frustración-satisfacción & 30 & .37 & 3 \\
\hline Promedio & 36 & .31 & 1.96 \\
\hline
\end{tabular}

Nota: Resultados obtenidos en el programa RECORD 5.0 (Elaboración propia).

La intervención ideal. Para esta terapeuta, llevar a cabo una intervención ideal en su práctica profesional, es de suma importancia la integración familiar, la empatía como piedra angular y el cuidado que debe tener como terapeuta en los sistemas en los cuales se interviene. Para ella, su fortaleza como terapeuta tiene estrecha relación con su disposición, cuidado y empatía hacia la familia.

Considera como importante para lograr los cambios deseados, la disposición de la familia con la que se trabaja. Así también, esta disponibilidad debe venir del terapeuta a cargo, es decir, si no existe la disposición y empatía del terapeuta para lograr adentrarse en el mundo de la familia, difícilmente se logra una intervención ideal u óptima. Para ella, la satisfacción que puede llegar a experimentar en su ejercicio profesional tiene relación con una intervención empática, de contención, siempre y cuando la disponibilidad sea recíproca.

Respecto a la familia con la que trabajó, nuevamente la empatía y la unión familiar, son valoradas como rasgo importante para el logro de objetivos, porque considera que el hecho de que los demás integrantes comprendan y logren ponerse en el lugar de su familiar mayor, puede generar la sensación de sentirse comprendido, valorado y escuchado y facilitar así una mejor percepción de calidad de vida, no solo personal sino también en las relaciones e integración familiar.

Para esta terapeuta las relaciones deberán ser los suficientemente fuertes para resistir el embate de la diabetes, fortaleza que se pondrá a prueba en los momentos de crisis de dicha enfermedad, pero que los puede hacer salir adelante como sistema familiar resiliente.

Resonancia de la interacción con un adulto mayor, en sus propios procesos de envejecimiento. Ahora bien, en cuanto a su propia historia de vida con un familiar con padecimiento diabético y a su proceso per- 
sonal de envejecimiento, puede valorarse como persona que empatiza, y a su vez cuida de un estilo de vida en el cual, la convivencia familiar y el cuidado personal son parte fundamental, y una manera efectiva de afrontar su proceso personal de envejecimiento. El reconocimiento y valoración de la familia de origen y su influencia, se convierte en un primordial recurso para vivir su propio proceso de envejecimiento de una manera saludable y con los cuidados necesarios.

Así también, al trabajar con estas familias valoró aspectos que por lo común cuando se es joven no se le da la importancia que merecen, sobre todo en lo referente a la salud como, tener la facilidad y destreza física de poder desplazarse a los lugares que se desea, el no depender de nadie para realizar las actividades, entre otros. El trabajar con familias que tienen a su cuidado un adulto mayor con padecimiento diabético, la ayudó a tomar conciencia sobre el cuidado que debe tener en su salud, alimentarse sanamente, dormir bien. Para poder envejecer con la mejor calidad de vida posible, pues lo importante no es vivir los más años que se puedan, si no envejecer con calidad.

\section{Resultados de la segunda terapeuta}

Constructos personales. Respecto a los resultados de la segunda terapeuta que se muestran en la tabla 2 , son los siguientes:

Índice de Polarización. Con puntuaciones más altas respecto al índice de polarización el cual hace referencia a la rigidez del constructo, fueron: Participación - no participar: 70; Sensible - cerrado: 60.; Empatía - no integrarse: 50; Buena - Irresponsable: 50; Placentero-enfadado: 50. Para la terapeuta, es importante mostrar empatía para realizar sus intervenciones y acompañamiento a la familia con un integrante con diabetes tipo 2 , en gran medida por los recursos que su familia de origen le ofrece, pues en ella se convive con un adulto mayor con dicho padecimiento. Por lo tanto, esto la hace incrementar su empeño por ser una buena terapeuta, que de acuerdo con sus constructos personales se definen por la empatía y sensibilidad al dolor de las personas que solicitan su atención, situación que hace de su quehacer terapéutico una manera placentera de realizarse, no solo en el aspecto personal sino en lo profesional.

Índice de Intensidad. Respecto al índice de intensidad el cual es indicador de la centralidad del constructo, se obtuvieron los siguientes resultados: Participación - no participar: .77; Empatía - no integrarse: .76; Sensible - cerrado: 75. Para ella es importante que su labor como terapeuta sea activa y participativa, no limitándose a ser un simple receptor del sufrimiento humano, por lo cual la sensibilidad, y la empatía también son constructos que estarían presentes.

Potencia Discriminativa. En lo concerniente a la potencia discriminativa la cual hace referencia a la flexibilidad en la aplicación del constructo, el resultado más alto es el concerniente a: Salud - enfermedad: 3 . Al saberse parte de un sistema familiar donde la diabetes está presente, puede tener un referente de los aspectos que ayudarían al adulto mayor a tener un mejor cuidado y calidad de vida si la familia colabora de manera activa, y también entiende que aún con un padecimiento crónico, se puede encontrar situaciones placenteras, sin que ello reduzca o vulnere el cuidado del adulto mayor, por eso esta experiencia que recupera de su familia de origen, es valorada como uno de sus mayores recursos al trabajar con este tipo de familias.

Intervención ideal. Para la segunda terapeuta, es de vital importancia la participación sensible en su práctica terapéutica para lograr optimizar los recursos de la familia, y lograr una intervención ideal. De igual manera, considera importante en su ejercicio profesional, la responsabilidad no solo de ella, sino también de la familia con un adulto mayor con diabetes tipo 2 en su propio cuidado, así como la empatía de los familiares sanos.

Las habilidades más destacables de esta terapeuta son la sensibilidad, disposición, responsabilidad en su ejercicio, empatía por los participantes de un contexto terapéutico, en el que ella se asume como un integrante más, donde su participación y sensibilidad, puede llevar a realizar lo que ella considera una intervención ideal.

Para ella, llevar a cabo una intervención ideal tiene que ver con el logro del cambio de los sistemas de interacción a interacciones más saludables en las familias con un adulto mayor con diabetes. Destaca el constructo participación, y lo define como benéfico para el éxito de la intervención ideal, pues gracias a que las 
Tabla 2.- Constructos e indicadores de la segunda terapeuta

\begin{tabular}{lccc}
\hline Constructos & Polarización & Intensidad & Potencia discriminativa \\
\hline Responsable-flojo & 40 & .74 & 2 \\
Soledad- estar conmigo & 40 & .63 & 2 \\
Empatía- no integrarse & $\mathbf{5 0}$ & $\mathbf{. 7 6}$ & 2 \\
Calidez- frialdad & 30 & .65 & 2 \\
Salud-enfermedad & 30 & .15 & $\mathbf{3}$ \\
Buena-irresponsable & $\mathbf{5 0}$ & .71 & 1.60 \\
Participación-no participar & $\mathbf{7 0}$ & $\mathbf{. 7 7}$ & 2 \\
Resistencia- adaptación & 20 & .68 & 2 \\
Sensible- cerrado & $\mathbf{6 0}$ & .75 & 1.5 \\
Placentero-enfadado & $\mathbf{5 0}$ & .73 & 1.5 \\
\hline Promedio & 44 & .41 & 1.32 \\
\hline
\end{tabular}

Nota: Resultados obtenidos en el programa RECORD 5.0 (Elaboración propia).

dos familias se interesaron en las actividades de las sesiones diseñadas, pudo vivirse desde el inicio hasta el final con interés y mucha correspondencia, consideración fundamental para un acercamiento real y auténtico, es decir, la experiencia se vive como un encuentro entre amigos más que como un trabajo clínico.

Por último, percibe como el mayor impacto y logro el nivel de sensibilidad alcanzado en cada familia, al comprender de manera empática lo que vive su familiar con este padecimiento, y enfatiza como medular el desarrollo de este proceso para que exista conciencia real y, por consiguiente, el surgimiento de manera natural y espontáneo de apoyar a su familiar.

Resonancia de la interacción con un adulto mayor, en sus propios procesos de envejecimiento. Para esta terapeuta, esta experiencia le brindó una gran enseñanza, porque al igual que la otra terapeuta, se identificó con su abuela materna que padece esta enfermedad. También en cuanto a su propio envejecimiento, aprendió a dar mayor importancia al cuidado psicológico (aminorar las situaciones estresantes, y realizar mayor actividad recreativa y física), ella misma se define como una persona sumamente responsable de su cuidado personal debido al antecedente de la diabetes en su familia, por lo que la responsabilidad no solo es puesta en práctica en su labor como terapeuta sino también en su proceso de envejecimiento personal, percibido como suficientemente sano para disminuir la predisposición genética de su familia.

\section{Conclusiones}

Al finalizar este estudio, logramos apreciar en ambas terapeutas, que los constructos personales atraviesan de manera importante las formas en que definen sus experiencias, tanto en sus familias de origen como en su práctica profesional, razón por la cual es importante que el terapeuta sea consciente de estos aspectos y formen parte de manera clara en la construcción de su estilo terapéutico, solo posible a través de un ejercicio dialógico reflexivo, para generar un andamiaje en el tránsito de lo familiar y conocido a lo posible por conocer (White, 2016). 
Para las terapeutas participantes, sus constructos personales son valores que se traducen en principios de vida, no se puede ser ellas mismas sin que éstos no estén presentes en cualquier ámbito en que interactúen, es lo subyacente de su actuación como personas y como terapeutas. A su vez, no podrían haber existido, sin la influencia del legado familiar, historia que nutre las resonancias tanto en su práctica profesional, al conectar con abuelas que viven o vivieron esta enfermedad crónica, como con ellas mismas al implementar mecanismos de prevención y hacia un proceso de envejecimiento saludable.

Por consiguiente, los constructos personales se tornan medulares, de manera que este trabajo clínico tuvo la posibilidad de ser catártico, desde la definición clásica, porque se convirtió en una experiencia emocional que movió a las terapeutas a un lugar de conexión con aquello que valoran y aprecian en sus vidas, fuertemente anclado en la historia de vida de su familia de origen, resignificando sus experiencias de vida y su práctica terapéutica (White, 2016: Estupiñán, 2005).

Finalmente, podemos constatar en este trabajo, que el encuentro de la familia y el terapeuta nunca es pasivo, siempre existe una transformación mutua, ser conscientes de ello es lo que enriquece y trasciende la teoría a una práctica real, auténtica y que no puede ser entendida en los libros. Con cada práctica e intervención que realizamos posibilitamos la creación de alternativas teóricas, de saberes que se dan en la interacción con los otros (Seguí, 2015; Estupiñán, 2005).

\section{Límites y recomendaciones}

Por ser un estudio de corte cualitativo, no podemos generalizar los resultados, suscribiéndose éstos exclusivamente a la experiencia de las participantes. Sin embargo, allí radica la riqueza del estudio, pues al enfocarse a la valoración del constructo personal, lo acerca a la persona misma, a su conocimiento local, al sujeto común y al auténtico experto en su vida.

Para futuros trabajos, se sugiere que, posterior al análisis de los constructos personales, se lleven a cabo entrevistas exploratorias en modalidad de equipo reflexivo, incluyendo a las familias con las que se realizó la intervención, con el afán de atestiguar el impacto que su experiencia de vida deja en los terapeutas y a su vez entablar reflexiones que puedan ser compartidas de tal forma que, sea evidente la influencia recíproca y constante de los sistemas familiares, es decir, la historia de la familia de origen del terapeuta como influyente en su práctica, e influida a la vez por las historias de las familias consultantes, y así honrar esos saberes y habilidades construidos de manera conjunta y que en ningún otro momento podría haber sido factible.

\section{Discusión}

La posibilidad de realizar una reflexión sobre las prácticas profesionales del terapeuta, a la luz de los fundamentos que la sostienen, pero además integrando el legado de saberes provenientes de su familia de origen, conforman un área de oportunidad en aras de la reconciliación, entre experiencia, teoría, práctica y saber técnico. La práctica auto reflexiva debería ser una acción continua y cuidadosa de toda creencia o práctica terapeútica, consciente de las posibles consecuencias que, de no hacerla tendría (Montagud, 2015).

Esta oportunidad que brinda el plano de la reflexividad sobre nuestras acciones y demás elementos presentes en nuestro trabajo clínico, posibilita la construcción de conocimiento y saber práctico, pero sobre todo la instancia de influir en intervenciones mucho más cercanas que las que encontramos en libros. Si bien es cierto que, esto se ha hecho desde una postura positivista, con estudios de largo alcance, es necesario desplegar el telón a los acercamientos de otras posturas metodológica como son las cualitativas, que abonan a la heterogeneidad y creatividad investigativa, teniendo como resultados una manera diferente de mirar la realidad, tal es el caso que propusimos en este trabajo, realizar una reflexión desde y sobre la acción de la actuación profesional del terapeuta, como estrategia y herramienta para mejorar sus abordajes clínicos (Catalán y Escobar, 2018; Canevaro, et al., 2016). 
Esta capacidad de hacer consciente dicho proceso y poder compartirlo con otros, permite la adquisición de nuevas posibilidades y alternativas en las formulaciones de soluciones contextualizadas a dimensiones sociales, históricas y culturales a problemas específicos, a su vez, esta oportunidad de compartir y dialogar por medio de la autorreflexión, contribuye a esa búsqueda de autenticidad creativa que caracteriza el estilo terapéutico que en proceso continuo y evolutivo el terapeuta va construyendo (Páez, et al., 2018).

Esta construcción de estilo terapéutico tiene una interconexión que vincula esta capacidad reflexiva, como eje en este proceso que trasciende a un estilo de vida, filosofía o paradigma personal. ¿Acaso, en el espacio terapéutico se deja al margen o en la sala de espera nuestra propia historia? Por ello, la importancia de conocer e identificar los constructos personales que como personas construimos y a través de los cuales vemos y modelamos nuestra realidad, dando sentido a lo que observamos y experimentamos (Roa, 2014; Ceberio, Moreno y Des Champs, 2000).

Ahora bien, con lo referente a las resonancias que como terapeutas tenemos en nuestro actuar clínico, es crucial la articulación entre lo personal, nuestra historia, e intereses, y el sentido social significativo y responsable que le atribuimos a nuestro proceder, como al de los otros, estos otros incluyen a la propia familia. Estas resonancias posicionan al terapeuta como pieza clave, pues sus acciones son vistas como un proceso en construcción copartícipe con la familia y la propia familia de origen, por lo que es necesaria su clara y fuerte presencia y debe aparecer incluso en sus anotaciones, bitácoras o diarios, en el que se abra un espacio a esa autorreflexión, en una especie de ejercicio dialógico, que asuma la implicación social, ética y política que conlleva la práctica terapéutica (Dantas De Biagi y Fernando, 2018; Estupiñán, 2005; Garciandía, y Samper, 2004).

En la investigación cualitativa, a este ejercicio se le denomina auto etnografía, en ella el investigador expone de forma sensible y detallada el análisis de sus emociones, pensamientos, relaciones con la investigación, y decisiones interpretativas. En sí, se trata de una auto comprensión del propio investigador, para que ello le facilite la comprensión e interpretación del relato del otro. Por esta razón se hace aún más conveniente que el terapeuta realice esta práctica reflexiva, porque además de estar enriqueciendo y potencializando su estilo terapéutico, estaría contribuyendo a la conformación de una teoría de la intervención clínica (Blanco, 2017; Chase, 2015).

Por lo que para la vida del terapeuta, su experiencia personal, al trabajar con estas familias, ayudó no solo a revisar las resonancias personales, sino a ser parte activa de sus vidas una vez que reflexionan y reintegran en ellas los diversos significados sobre sus familiares mayores con diabetes, lo que contribuye no solo a la identificación sino al reconocimiento significativo de dichas personas como colaboradores y constructores en la reescritura de sus nuevas historias de vida personal y profesional, así como, de los saberes y habilidades que ahora adquirieron con respecto a la enfermedad crónica, estilos de vida saludable, y la conciencia de un buen proceso de envejecimiento en sus propias vidas (Szmulewicz, 2013; Ceberio y Linares, 2005).

\section{Referencias}

Andersen, T. (2005). Procesos de reflexión: actos informativos y formativos. En S. Friedman (Comp.), Terapia familiar con equipo de reflexión (pp. 39-69). Buenos Aires, Argentina: Amorrurtu.

Blanco, M. (2017). Investigación Narrativa y Autoetnografía: Semejanzas y Diferencias. Investigación Cualitativa, 2(1), 66-80. https://doi.org//10.23935/2016/01037

Canevaro, A., Abeliuk, Y., Condeza, M., Escala M. A., García, G. y Montes, J. (2016). El nacimiento del terapeuta sistémico. El trabajo directo con las familias de origen de los terapeutas en formación, Revista REDES de Psicoterapia Relacional e Intervenciones Sociales. Recuperado de http://redesdigital.com.mx/index.php/ redes/article/view/173/131

Catalán M. M., y Escobar S. M. (2018). Una experiencia de innovación a través de la incorporación del aprendizaje reflexivo en la práctica clínica en comunidad, como estrategia para integrar las competencias actitudinales en la formación profesional de los estudiantes de enfermería, Universidad Pedro de Valdivia (Tesis doctoral). Universidad Andrés Bello. Recuperada de http://repositorio.unab.cl/xmlui/handle/ria/7240

Ceberio, M. y Linares, J. L. (2005). Ser y hacer en terapia sistémica: La construcción del estilo terapéutico. Barcelona, España: Paidós. 
Ceberio, M. Moreno, J., y Des Champs, C. (2000). La formación y el estilo del terapeuta, Revista Mosaico, 3(60), 1-24.

Chase, S. E. (2015). Investigación narrativa. Multiplicidad de enfoques, perspectivas y voces. En N. Denzin, y Y. S. Lincoln, (Comp.) Manual de investigación cualitativa. (Vol. IV, pp. 59-112). Buenos Aires, Argentina: Editorial Gedisa.

Dantas De Biagi, A. B. y Fernando, R. E. (2018). A construção da responsabilidade relacional em terapia familiar. Pensando Familias, 22(1), 3-17.

Denzin, N. K y Lincoln, Y. S. (2015). Manual de investigación cualitativa.(Vol. IV). Buenos Aires, Argentina: Editorial Gedisa.

Estupiñán, M. J. (2005). Psicoterapia sistémica, psicología y responsabilidad social: la hipótesis de la convergencia entre sabiduría y conocimiento técnico. Diversitas: Perspectivas en Psicología, 1, 227-237. https:// doi.org/10.15332/s1794-9998.2005.0002.10

Feixas, G. y Cornejo, J. M. (1996). Manual de la técnica de rejilla. Barcelona, España: Paidós.

Framo, J. (1996). La Familia de origen y psicoterapia: un enfoque intergeneracional. Barcelona, España: Paidós.

Garciandía, J. A. y Samper, J. (2004). El tejido de un nosotros: hilando nuevos significados entre terapeuta y consultante. Revista Colombiana de Psiquiatría, 33, 263-284.

Hernández, C. A. (2012). Vínculos, individuación y ecología humana, hacia una psicología clínica compleja. Revista Latinoamericana de Ciencias Sociales, Niñez y Juventud, 10(1), 687-689.

Hernández, R. Fernández, C. Baptista, M. (2014). Metodología de la investigación. Ciudad de México, México: Mc Graw Hill.

Kelly, G. A. (1955). The psychology of personal constructs (2 Vols.). Nueva York, Estados Unidos: Norton.

Latorre, A., Del Rincón, D., Arnal, J. (1996). Bases Metodológicas de la investigación educativa. Barcelona, España: Hurtado Ediciones.

Louro, B. I. (2003). La familia en la determinación de la salud. Revista Cubana Salud Pública, 29(1), 48-51.

Montagud, M. X. (2015). Complejidad, reflexividad y autoetnografía. Las posibilidades de la investigación narrativa en la mejora de la práctica profesional. Trabajo Social Global, 5(9), 3-23.

Neimeyer, G. (1996). Evaluación constructivista. Buenos Aires, Argentina: Paidós.

Neimeyer, R. y Mahoney, M. (1998). Constructivismo en psicoterapia. Barcelona, España: Paidós.

Páez, M. L., Arcila, A., Cabiedes, J. S., Cardona, C., Correa, D., Guevara, V. (2018). Formación de terapeutas sistémicos: desde el saber teórico y práctico hacia la autorreferencia y la construcción del estilo terapéutico. Tempus Psicológico, 1(1), 13-38. https://doi.org/10.30554/tempuspsi.1.1.1992.2018

Pakman, M. (1999). Investigación e Intervención en Grupos Familiares. Una Perspectiva Constructivista. En J. M. Delgado y J. Gutiérrez (Eds.), Métodos y técnicas cualitativas de investigación en ciencias sociales (pp. 359-377). Madrid, España: Editorial Síntesis.

Roa, A. M. B. (2014). Movilización de los aprendizajes de terapeutas en formación que surgen de las conversaciones reflexivas a partir de la emergencia del observador en el contexto de supervisión. Quaestiones Disputatae: Temas en Debate, 7(14), 67-80.

Seguí, D. J. (2015). Mentalidad Humana. Barcelona, España: Endiálogo ediciones.

Szmulewicz, E. T. (2013). La persona del terapeuta: eje fundamental de todo proceso terapéutico. Revista Chilena de Neuropsiquiatría, 51(1), 61-69. https://doi.org//10.4067/S0717-92272013000100008

White, M. (2002). El enfoque narrativo en la experiencia de los terapeutas. Barcelona, España: Gedisa. White, M. (2011). Práctica narrativa . La conversación continúa. Santiago, Chile: Pranas Chile ediciones. White, M. (2016). Mapas de la práctica narrativa. Santiago, Chile: Pranas Chile ediciones.

Artículo recibido: $27 / 05 / 2018$

Artículo aceptado: 22/10/2018 